KCL-TH-97-54

hep-th/9710033

September 1997

\title{
The Threebrane Soliton of the M-Fivebrane
}

\author{
P.S. Howe \\ N.D. Lambert \\ and \\ P.C. West ${ }^{\star}$ \\ Department of Mathematics \\ King's College, London \\ England \\ $W C 2 R$ 2LS
}

\begin{abstract}
We discuss the supersymmetry algebra of the $\mathrm{M}$ theory fivebrane and obtain a new threebrane soliton preserving half of the six-dimensional supersymmetry. This solution is dimensionally reduced to various $\mathrm{D}-p$-branes.
\end{abstract}

^ phowe, lambert, pwest@mth.kcl.ac.uk 


\section{Introduction}

The dynamics of the $\mathrm{M}$ theory fivebrane are given by an interacting $(2,0)$ tensor multiplet containing a self-dual three tensor $h_{m n p},(m, n, p=0, \ldots, 5)$ five scalars $X^{b^{\prime}}\left(b^{\prime}=1^{\prime}, \ldots, 5^{\prime}\right)$ and sixteen fermions $\Theta_{\alpha}^{i},(\alpha=1, \ldots, 4, i=1, \ldots 4)$. In this paper we shall use the formalism and notations of [1] but there are also other formulations available [2]. In this short paper we wish to examine some properties of the supersymmetric soliton states in this theory.

The most general form for the $(2,0)$ supersymmetry algebra in six dimensions is

$$
\left\{Q_{\alpha}^{i}, Q_{\beta}^{j}\right\}=\eta^{i j}\left(\gamma^{m}\right)_{\alpha \beta} P_{m}+\left(\gamma^{m}\right)_{\alpha \beta} Z_{m}^{i j}+\left(\gamma^{m n p}\right)_{\alpha \beta} Z_{m n p}^{i j}
$$

Here $\eta^{i j}$ is the invariant tensor of $U S p(4) \cong \operatorname{Spin}(5), P_{m}$ is the momentum, $Z_{m}^{i j}$ is in the symmetric 5 of $\operatorname{Spin}(5)$ and $Z_{m n p}^{i j}$ is self-dual and in the anti-symmetric 10 of $\operatorname{Spin}(5)$. It is possible to add to (1.1) a five form central charge in the $\mathbf{5}$ of $\operatorname{Spin}(5)$, however, due to the self-duality constraint it contains no additional degrees of freedom. In fact the left hand side can be thought of as a sixteen by sixteen symmetric matrix and therefore has $\frac{16 \times 17}{2}=136$ degrees of freedom. Similarly the right hand side contains $6+5 \times 6+10 \times 10=136$ degrees of freedom and hence there can be no additional independent terms [3]. Since the $p$-form charges of $p$-dimensional extended objects can give rise the additional central charges in (1.1) [4], we may expect to find onebrane and threebrane solitons of the M-fivebrane equations preserving half of the spacetime supersymmetry. We will shortly return to the interpretation of the five form central charge.

There are in fact two other arguments which also lead to the appearance of onebranes and threebranes on the M-fivebrane worldvolume. The first is from the $\mathrm{M}$ theory interpretation in eleven dimensions. Here one has two possible configurations which preserve a quarter of the eleven-dimensional supersymmetry; a membrane intersecting a fivebrane over a onebrane [5] or two fivebranes intersecting over a threebrane [6]. In terms of the worldvolume of the fivebrane these config- 
urations will appear as onebrane and threebrane solitons respectively, preserving half of the six-dimensional supersymmetry.

Another reason one may expect only one form and three form central charges comes from the interpretation of these configurations as the effective field outside of a D-p-brane [7] of the self-dual string theory. Here we will assume that the notion of a D-p-brane (as a $(p+1)$-dimensional hyperplane in spacetime where open strings end) can be extended to an analogous object of the six-dimensional self-dual string theory. In this case the supersymmetries preserved by the modified boundary conditions on the self-dual string are those for which

$$
\epsilon_{L}=\Gamma_{0} \ldots \Gamma_{p} \epsilon_{R}
$$

where the D-brane lies in the $x^{0}, \ldots, x^{p}$ plane and $\epsilon_{L}$ and $\epsilon_{R}$ are the left and right handed supersymmetry generators on the self-dual string worldsheet respectively. Since $\epsilon_{L}$ and $\epsilon_{R}$ are of the same six-dimensional chirality it is easy to check that (1.2) is consistent if and only if $p=1,3,5$. In this case there again appears the possibility of a D-fivebrane on the worldvolume and hence a five form central charge in (1.1). This would be analogous the the D-ninebrane of the type IIB string and carry no physical degrees of freedom. It does however point to the possibility of performing an orientifold projection to a self-dual $(1,0)$ string theory in six dimensions, in analogy with the construction of the type I string as an orientifold of the type IIB string [7]. The analogy with type IIB string theory also suggests that the six-dimensional self-dual string may possess a sort of D-instanton or -1-brane. However we shall see below that this is not the case.

This $(1,0)$ string theory can be understood within $M$ theory if we suppose that there is some kind of ninebrane. It would then be possible for a ninebrane to intersect a fivebrane over a fivebrane (i.e. a fivebrane-ninebrane bound state). This would give rise to a five form central charge on the fivebrane. The ninebrane

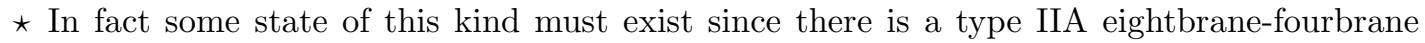
bound state preserving eight supersymmetries, indeed we shall construct it below.
} 
would then have four directions transverse to the fivebrane, indicated by four of the five scalars. By choosing which four scalars of the five on the worldvolume to use one obtains a multiplet of fivebranes in the $\mathbf{5}$ of $\operatorname{Spin}(5)$, in agreement with the algebra (1.1). This construction of the $(1,0)$ self-dual string has appeared in [8], in connection with the fivebrane of the $E_{8} \times E_{8}$ heterotic string.

In [9] a family of supersymmetric onebrane solitons on the M-fivebranes equations of motion were found, transforming under the $\mathbf{5}$ of $\operatorname{Spin}(5)$. In this paper we

shall seek a family of supersymmetric threebrane solitons in the $\mathbf{1 0}$ of $\operatorname{Spin}(5)$ and thereby complete the $p$-brane spectrum of the M-fivebrane. The threebrane of the M-fivebrane is also of some interest in its own right due to its relation to the low energy Seiberg-Witten effective action of $N=2, D=4$ Yang-Mills theory [10]. The precise relation between the dynamics of the two is the subject of a forthcoming paper [11]. In the next section we will obtain multi-threebrane solutions and describe their elementary properties. In the final section we will use the threebrane solitons to obtain $(p-1)$-branes and $(p-2)$-branes on the D-p-branes of type II string theory.

\section{The Threebrane Soliton}

In this paper we denote the six-dimensional coordinates of the fivebrane by hatted variables $\hat{m}, \hat{n}=0,1,2, \ldots, 5$. In addition all indices are raised and lowered with respect to the flat Euclidean metric unless explicitly indicated otherwise. Let us look for a threebrane in the plane $x^{0}, x^{1}, x^{2}, x^{3}$. We let unhatted variables refer to the transverse coordinates of the threebrane i.e. $x^{4}, x^{5}$. We will assume all fields to depend only on the transverse coordinates. To find a threebrane we dualise one scalar $X^{1^{\prime}}$ to a five-form $G_{5}$

$$
G_{\hat{m} \hat{n} \hat{p} \hat{q} \hat{r}}=\epsilon_{\hat{m} \hat{n} \hat{p} \hat{q} \hat{r} \hat{s}} \partial^{\hat{s}} X^{1^{\prime}}
$$


Our ansatz is then

$$
\begin{aligned}
G_{0123 m} & =\epsilon_{m n} \partial^{n} X^{1^{\prime}} \equiv v_{m} \\
X^{2^{\prime}} & =\phi \\
h_{m n p} & =0
\end{aligned}
$$

where $\epsilon_{m n}$ is the volume element on the transverse space and the other scalars $X^{3^{\prime}}, X^{4^{\prime}}, X^{5^{\prime}}$ are constant. With this ansatz the equations of motion of the fivebrane simplify greatly. We introduce the metric

$$
g_{\hat{m} \hat{n}}=\left(\begin{array}{ccc}
-1 & 0 & \\
0 & \mathbf{1}_{3 \times 3} & \\
& & \delta_{m n}+\partial_{m} X^{1^{\prime}} \partial_{n} X^{1^{\prime}}+\partial_{m} X^{2^{\prime}} \partial_{n} X^{2^{\prime}}
\end{array}\right)
$$

where $\mathbf{1}_{3 \times 3}$ this the unit matrix in three dimensions. Since the three form is zero the only equations of motion we need to solve for are [1]

$$
g^{m n} \nabla_{m} \nabla_{n} X^{1^{\prime}}=g^{m n} \nabla_{m} \nabla_{n} X^{2^{\prime}}=0
$$

where $\nabla$ is the Levi-Civita connection of $g_{\hat{m} \hat{n}}$.

As with the string soliton found in [9] it is helpful now to determine the condition that half of the supersymmetry is preserved by the soliton. It is instructive to first consider the linearised supersymmetry

$$
\begin{aligned}
\delta_{0} \Theta_{\beta}^{j} & =\epsilon^{\alpha i}\left(\frac{1}{2}\left(\gamma^{m}\right)_{\alpha \beta}\left(\gamma_{b^{\prime}}\right)_{i}^{j} \partial_{m} X^{b^{\prime}}-\frac{1}{6}\left(\gamma^{m n p}\right)_{\alpha \beta} \delta_{i}^{j} h_{m n p}\right) \\
& =\frac{1}{2} \epsilon^{\alpha i}\left(\gamma^{m}\right)_{\alpha \beta}\left(\gamma_{b^{\prime}}\right)_{i}^{j} \partial_{m} X^{b^{\prime}}
\end{aligned}
$$

for $h_{m n p}=0$. Inserting our ansatz in to $\delta_{0} \Theta_{\beta}^{j}=0$ one finds

$$
\begin{aligned}
0=\frac{1}{2} \epsilon^{\alpha i}\left(\gamma^{4}\right)_{\alpha \gamma}\left(\gamma_{1^{\prime}}\right)_{i}{ }^{k} & \left\{\left[\delta_{k}^{j} \delta_{\beta}^{\gamma} v_{5}+\left(\gamma_{1^{\prime} 2^{\prime}}\right)_{k}^{j}\left(\gamma^{45}\right)_{\beta}^{\gamma} \partial_{5} \phi\right]\right. \\
& \left.+\left(\gamma_{1^{\prime} 2^{\prime}}\right)_{k}^{l}\left[\delta_{l}^{j} \delta_{\beta}^{\gamma} \partial_{4} \phi+\left(\gamma_{1^{\prime} 2^{\prime}}\right)_{l}^{j}\left(\gamma^{45}\right)_{\beta}^{\gamma} v_{4}\right]\right\} .
\end{aligned}
$$


Thus if we set

$$
v_{m}= \pm \partial_{m} \phi
$$

we find that the solution will be invariant under supersymmetries which satisfy

$$
\epsilon_{0}^{\alpha i}\left(\gamma_{1^{\prime} 2^{\prime}}\right)_{i}{ }^{j}\left(\gamma^{45}\right)_{\alpha}^{\beta}=\mp \epsilon_{0}^{\beta j}
$$

Note that the bogomol'nyi condition (2.7) is equivalent to the statement that $X^{1^{\prime}}+$ $i X^{2^{\prime}}$ is an (anti-)holomorphic function of $x^{4}+i x^{5}$ for the minus (plus) sign in (2.7). To verify that the supersymmetries are preserved to all orders we recall the expression obtained in [9] for the full non-linear supersymmetry

$$
\delta \Theta_{\beta}^{j}=-\frac{1}{2} \frac{1}{\sqrt{-\operatorname{det} g}} \epsilon^{\alpha i}\left(E^{-1}\right)_{\alpha i}{ }^{\gamma k} \partial_{m} X^{b^{\prime}}\left(\gamma^{m}\right)_{\gamma \delta}\left(\gamma_{b^{\prime}}\right)_{k}^{l}(u)_{l \beta}^{\delta}{ }_{l j}
$$

Here we have set $h_{m n p}=0$ and we refer the reader to [9] for a detailed description of the matrices $E$ and $u$. Clearly (2.9) vanishes if $\delta_{0} \Theta_{\beta}^{j}=0$, provided that the preserved supersymmetries are $\epsilon^{\beta j}=\epsilon_{0}^{\alpha i} E_{\alpha i}{ }^{\beta j}$, where $\epsilon_{0}^{\alpha i}$ satisfies (2.8).

If we substitute the Bogomol'nyi condition (2.7) into the metric (2.3) it is now an easy matter to see that the equation of motion becomes

$$
\frac{1}{1+(\partial \phi)^{2}} \partial^{2} \phi=0
$$

where $\partial^{2} \phi=\delta^{m n} \partial_{m} \partial_{n} \phi$ and $(\partial \phi)^{2}=\delta^{m n} \partial_{m} \phi \partial_{n} \phi$. Furthermore it follows from the Bogomoln'yi condition (2.7), that (2.10) is solved by any $X^{1^{\prime}}, X^{2^{\prime}}$. One can also check from (2.10) that $G_{5}$ is a closed form. Thus the solution corresponding to $N$ threebranes located at $y_{I}(I=0, \ldots, N-1)$ has the general form

$$
\begin{aligned}
G_{0123 m} & = \pm \partial_{m} \phi, \\
\phi & =\phi_{0}+\sum_{I=0}^{N-1} Q_{I} \ln \left|x-y_{I}\right|,
\end{aligned}
$$

where $\phi_{0}$ and $Q_{I}$ are constants. Clearly this solution has bad asymptotic behaviour 
unless $\sum Q_{I}=0$, however one can still define the charge of a single threebrane

$$
Q=\frac{1}{2 \pi} \int_{S_{\infty}^{1}} \star G_{5}= \pm Q_{0}
$$

where $S_{\infty}^{1}$ is the transverse circle at infinity and $\star$ is the flat six-dimensional Hodge star. The presence of the conformal factor in (2.10) indicates that the equations of motion are satisfied even at the points where the solution is ill behaved and hence no sources are needed.

Let us consider the zero modes of a single threebrane soliton. Clearly there are two bosonic zero modes $y_{0}^{m}$ describing the location of the threebrane in the transverse space. Now consider the three-form $h_{3}=d b_{2}=0$. The closed two form $b_{2}$ has a gauge symmetry $b_{2} \rightarrow b_{2}+d A_{1}$. However, because of Poincaré invariance of the threebrane along $x^{0}, x^{1}, x^{2}, x^{3}$, there are vector fields $A_{1}$ whose indices tangent to the threebrane do not vanish at infinity, corresponding to socalled large gauge transformations. Following the standard treatment of soliton zero-modes (see for example [12]), we must interpret these components of $A_{1}$ as zero modes of the threebrane. Thus there is also a four dimensional vector zero mode living on the threebrane worldvolume* The fermionic zero modes come from the broken supersymmetries and hence there are eight of them and in four dimensions they are necessarily non-chiral. Thus we find an $N=2, D=4$ vector multiplet of zero modes on the threebrane.

Since the scalars transform under Spin(5), by choosing an arbitrary pair of scalars $\left(X^{a^{\prime}}, X^{b^{\prime}}\right)$ with $a^{\prime} \neq b^{\prime}$ for our solution, we obtain a multiplet of threebranes transforming as a $\frac{5 \times 4}{2}=10$ dimensional representation of $\operatorname{Spin}(5)$, in agreement with the algebra (1.1). In the $\mathrm{M}$ theory interpretation, where the threebrane represents the intersection of two fivebranes, these scalars point along the two

\footnotetext{
* The same reasoning also implies that there is a two dimensional vector zero mode on the self-dual string soliton worldvolume. This was ignored in [9] because it carries no degrees of freedom.
} 
directions of the external fivebrane which are transverse to the worldvolume of the fivebrane we are considering.

We can also see that there is no BPS instanton configuration or -1-brane. For in this case we would again have only two non-trivial scalars, $X^{1^{\prime}}$ and $X^{2^{\prime}}$, with $\partial_{m} X^{1^{\prime}}$ interpreted as the field strength of the -1 -brane. However, what made the threebrane supersymmetric was the fact that in the two-dimensional transverse space the two scalars (or more precisely their field strengths) are dual to each other. For a -1-brane, where the transverse space is the six-dimensional Euclidean worldvolume of the fivebrane, there is no such condition. In fact one could always perform a Spin(5) rotation to a configuration with just one scalar. Clearly such a configuration could not preserve any supersymmetry.

\section{Discussion}

In this paper we have discussed the one half supersymmetric BPS states of the M theory fivebrane and in particular we obtained a new threebrane soliton. We also determined the zero modes of resulting $p$-branes. We note that the zero modes can be obtained by the dimensional reduction of a $D=6, N=(1,0)$ super-Maxwell multiplet to $p+1$ dimensions. We would like to conclude with a brief discussion of the dimensional reduction of the threebrane to D-p-brane worldvolumes.

It was shown in [1] that the fivebrane equations of motion can be dimensionally reduced to those of the D-fourbrane. Thus the threebrane soliton can be double dimensionally reduced to a twobrane on the D-fourbrane. By T-duality these can be extended to $(p-2)$ solitons on a D-p-brane (we will restrict our attention to $p \geq 2)$. These $(p-2)$-branes have exactly the same form as (2.11), only with $G_{5}$ now replaced by a $p$-form $G_{0 \ldots(p-2) m}= \pm \partial_{m} \phi$. These solutions represent two intersecting $\mathrm{D}$ - $p$-branes over a $(p-2)$-brane.

The above discussion only works for $p<8$ since the D-eightbrane only has one scalar field on its worldvolume. Therefore the naive extrapolation of the soliton (2.11) which involves two scalars cannot work. Instead the vector field on the 
eightbrane must become non-zero. More precisely consider two D-fourbranes intersecting over a twobrane (say one in the $x^{0}, x^{1}, x^{2}, x^{3}, x^{4}$ plane and the other in the $x^{0}, x^{1}, x^{2}, x^{5}, x^{6}$ plane). Now T-dualise along $x^{5}, x^{6}, x^{7}, x^{8}$ to obtain an eightbrane in the $x^{0}, \ldots, x^{8}$ plane, containing a fourbrane in the $x^{0}, x^{1}, x^{2}, x^{7}, x^{8}$ plane. We expect that this configuration is actually described by an instanton in the four transverse dimensions to the fourbrane; $x^{3}, x^{4}, x^{5}, x^{6}$. To see this explicitly let us construct the vector field $A_{m}$ in this transverse space. Now, in order to have applied T-duality we must set $A_{5}=A_{6}=0$ (or at least they must be pure gauge). Consequently, if we reinterpret $A_{3}=X^{1^{\prime}}$ and $A_{4}=X^{2^{\prime}}$, we find that the Bogomol'nyi condition (2.7) (i.e. holomorphicity) is equivalent to self-duality of the gauge field $A_{m}$.

One could also directly dimensionally reduce the threebrane soliton (2.11) to a threebrane on the D-fourbrane worldvolume. This leads to a domain wall on the fourbrane and is associated with a cosomological constant, which can be obtained by a Sherk-Schwarz type of dimensional reduction invoking one of the scalars. The form of this solution should also be clear to the reader, the only modification being that there is only one transverse dimension so that the logarithms in (2.11) are replaced by linear functions. This threebrane soliton represents a D-fourbrane intersecting with an NS-fivebrane. Clearly this solution can also be T-dualised to other D-p-branes intersecting with an NS-fivebrane over a $(p-1)$-brane.

Similarly to the above case this only works for $p<7$. To obtain a sevenbrane one must T-dualise in the transverse space of the NS-fivebrane. This leads to a Kaluza-Klein like fivebrane (i.e. a geometry which is $\mathbf{R}^{6} \times M_{T a u b-N U T}$ ), bound to the sevenbrane, which is wrapped around one compact and one non-compact dimension of $M_{\text {Taub-NUT }}$. It is impossible to T-dualise again to obtain an eightbrane configuration, since there is no suitable Taub-Nut geometry with the (local) form $\mathbf{R}^{2} \times \mathbf{T}^{2}$. This is reflected by the fact that there is no way to obtain a cosmological constant in the nine-dimensional Maxwell action by performing a Sherk-Schwarz reduction from ten dimensions. 
We would like to thank G. Papadopolous and H.A. Chamblin for discussions.

\section{REFERENCES}

1. P.S. Howe, E. Sezgin and P.C. West, Phys. Lett. B399 (1997) 49, hepth/9702008

2. M. Perry and J.H. Schwarz, Nucl. Phys. B489 (1997) 47, hep-th/9611065; M. Aganagic, J. Park, C. Popescu, and J. H. Schwarz, Worldvolume action of the M-theory fivebrane, hep-th/9701166; I. Bandos, K Lechner, A. Nurmagambetov, P. Pasti and D. Sorokin, and M. Tonin, Covariant action for the super fivebrane of M-theory, hep-th/9701149

3. P.K. Townsend, P-brane Democrary, hep-th/9507048

4. J.A. de Azcarraga, J.P. Gauntlett, J.M. Izquierdo and P.K. Townsend, Phys. Rev. Lett. 63 (1989) 2443

5. A. Strominger, Phys. lett. B383 (1995) 44, hep-th/9512059

6. G. Papadopoulos and P.K. Townsend, Phys, Lett. B384 (1996) 86, hepth/9605146

7. J. Polchinski, Phys. Rev. Lett. 75 (1995) 4724, hep-th/9510017

8. O. Ganor and A. Hanany, Nucl. Phys. B474 (1996) 122, hep-th/9602120

9. P.S. Howe, N.D. Lambert and P.C. West, The Self-Dual String Soliton, hep-th/9709014

10. E. Witten, Solutions of Four Dimensional Field Theories via M Theory, hep-th/97003166

11. P.S. Howe, N.D. Lambert and P.C. West, Classical M-Fivebrane Dynamics and Quantum $N=2$ Yang-Mills, KCL-TH-97-55

12. J.A. Harvey, Magnetic Monopoles, Duality and Supersymmetry, hepth/9603086 